\title{
Fine Mapping a Broad-Spectrum Powdery Mildew Resistance Gene in Chinese Landrace Datoumai, PmDTM, and Its Relationship with Pm24
}

\author{
Nan Lu, ${ }^{1,2}$ Mingxue Lu, ${ }^{1}$ Pan Liu, ${ }^{1}$ Hongxing Xu, ${ }^{1}$ Xiaolong Qiu, ${ }^{1}$ Shanshan Hu, ${ }^{1}$ Yanan Wu, ${ }^{1}$ Shenglong Bai, ${ }^{1}$ Jizhong Wu, ${ }^{3}$ \\ and Shulin Xue $\mathrm{X}^{1, \dagger}$ \\ ${ }^{1}$ State Key Laboratory of Crop Stress Adaptation and Improvement, State Key Laboratory of Cotton Biology, School of Life \\ Sciences, Henan University, Kaifeng 475004, Henan, China \\ ${ }^{2}$ Applied Plant Genomics Laboratory, Crop Genomics and Bioinformatics Centre, Nanjing Agricultural University, Nanjing \\ 210095, Jiangsu, China \\ ${ }^{3}$ Institute of Germplasm Resources and Biotechnology, Jiangsu Academy of Agricultural Sciences, Nanjing 210014, Jiangsu, \\ China
}

\begin{abstract}
Powdery mildew, caused by the biotrophic fungal pathogen Blumeria graminis f. sp. tritici $(B g t)$, is a globally important wheat disease causing severe yield losses, and deployment of resistant varieties is the preferred choice for managing this disease. Chinese wheat landrace Datoumai was resistant to 22 of $23 \mathrm{Bgt}$ isolates at the seedling stage. Genetic analysis based on the inoculation of $B g t$ isolate $\mathrm{E} 09$ on the $\mathrm{F}_{1}, \mathrm{~F}_{2}$, and $\mathrm{F}_{2: 3}$ populations derived from the cross Datoumai $\times$ Huixianhong revealed that the powdery mildew resistance of Datoumai is controlled by a single dominant gene, temporarily designated as PmDTM. Bulked segregant analysis and simple sequence repeat mapping with $200 \mathrm{~F}_{2}$ plants showed that PmDTM was located in the same genetic region as Pm24 on chromo-

$38,908,656 \mathrm{bp})$ on chromosome 1DS, and only one highly confident gene, TraesCS1D02G058900, was annotated within this region. TraesCS1D02G058900 encodes a receptor-like serine/threonine-protein kinase (STK), and a 6-bp deletion in exon 5 may confer the resistance to powdery mildew. Allele frequency analysis indicated that the STK allele with 6-bp deletion was only present in three landraces (Datoumai, Chiyacao [Pr24], and Hulutou) and was absent in all of the 353 Chinese modern cultivars and 147 foreign cultivars. These results demonstrate that PmDTM is mapped to the same locus as Pm24 and can be widely used to enhance powdery mildew resistance in wheat growing regions worldwide.
\end{abstract} some 1DS. To fine map PmDTM, 12 critical recombinants were identified from $1,192 \mathrm{~F}_{2}$ plants and delimited PmDTM to a 0.5-cM Xhnu58800 to Xhnu59000 interval covering $180.5 \mathrm{~Kb}(38,728,125$ to
Keywords: Blumeria graminis f. sp. tritici, candidate gene, fine mapping, powdery mildew, $P m 24$, wheat
Bread wheat (Triticum aestivum L.) is one of the most important crops and a staple food for about $40 \%$ of the world population. Wheat yield is constantly challenged by many diseases, and wheat powdery mildew, caused by the fungus Blumeria graminis (DC.) Speer $\mathrm{f}$. sp. tritici emend. É. J. Marchal (Bgt), is an epidemic foliar disease worldwide, and the pathogen competes for nutrients and reduces the photosynthetic ability of the leaves (Yao et al. 2007). The use of high-yielding dwarf varieties and modern wheat cultivation technologies have further increased the epidemic areas of powdery mildew (Singh et al. 2016). In China, powdery mildew has affected an area of around 6 to 8 million hectares in recent years (Sun et al. 2018 ). Both yield and quality of wheat suffer seriously from powdery mildew, and the commercial yield losses are 30 to $40 \%$ in heavy epidemic years (Conner et al. 2003; Samobor et al. 2006; Singh et al. 2016). Genetically controlled resistance is the basis of breeding for powdery mildew-resistant cultivars, which is critical for combating this disease.

${ }^{\dagger}$ Corresponding author: S. Xue; xsl@ vip.henu.edu.cn

Funding: This study was partially supported by National Natural Science Foundation of China (31971888), Key Technology R\&D Program of Henan Province of China (192102110013), Jiangsu Agricultural Science and Technology Innovation Fund of China (CX [17] 3004), and Jiangsu Collaborative Innovation Center for Modern Crop Production.

*The $\boldsymbol{e}$-Xtra logo stands for "electronic extra" and indicates there are supplementary materials published online.

The author(s) declare no conflict of interest.

Accepted for publication 20 January 2020.

(C) 2020 The American Phytopathological Society
Two types of powdery mildew resistance, qualitative and quantitative resistance, have been reported in wheat (Huang and Röder 2004). Qualitative resistance, controlled by single genes, is most widely employed in wheat breeding programs. Up to now, 65 permanently designated genes and over 20 temporarily designated $P m$ or $M l$ resistance genes or quantitative trait loci have been identified (Li et al. 2019a; McIntosh et al. 2013, 2017). However, due to the complexity of wheat genome (15.4 to $15.8 \mathrm{~Gb}$, IWGSC 2018), only seven genes (Pm2, Pm3, Pm8, Pm21, Pm38, Pm46, and Pm60) have been cloned to date (He et al. 2018; Hurni et al. 2013; Krattinger et al. 2009; Moore et al. 2015; Sánchez-Martín et al. 2016; Yahiaoui et al. 2004; Zou et al. 2018). Among them, Pm3, Pm21, $P m 38, P m 46$, and $P m 60$ were isolated by standard map-based cloning. These cloned powdery mildew resistance genes can be divided into two categories. Genes of the first category, such as $P m 2, P m 3$, $P m 8, P m 21$, and Pm60, confer race-specific resistance and generally encode a nucleotide-binding and leucine-rich repeat (NBLRR)-type protein that activates effector-triggered immunity. Genes of the second category such as $P m 38$ (Krattinger et al. 2009) and Pm46 (Moore et al. 2015), confer non-race-specific resistance or adult-plant resistance and encode an ATP-binding cassette transporter or a hexose transporter. The second category genes often provide durable resistance to multiple diseases including powdery mildew, leaf rust, and stripe rust. In addition to these two resistance mechanisms, the plant receptor-like protein kinase superfamily consisting of transmembrane proteins also plays important roles in plant defense (Liu et al. 2019). These receptors recognize microbial-associated or pathogen-associated molecular patterns (PAMPs) and activate PAMP-triggered immunity (Jones and Dangl 2006). For example, wheat gene Yr15, comprising two tandem kinase-pseudokinase domains, provides broad-spectrum resistance against stripe rust (Klymiuk et al. 2018). However, the protein kinase genes involved in powdery mildew resistance in wheat have not been cloned and reported to date. 
The rapid emergence of virulent $B g t$ isolates has prompted the search for more durable resistance. Broad-spectrum resistance genes are much sought after by breeders, because such genes generally can provide robust protection against diseases. The majority of the mapped genes with broad-spectrum powdery mildew resistance come from wild species or wheat relatives, such as $\mathrm{Pm} 12$ from Aegilops speltoides (Jia et al. 1996), Pm16 from wild emmer (Chen et al. 2005), Pm20 from rye (Friebe et al. 1994), and Pm21 and Pm62 from Dasypyrum villosum (He et al. 2018; Zhang et al. 2018). However, no elite cultivars with these genes except $P m 21$ have been developed due to the unfavorable linkage drag (Summers and Brown 2013). On the other hand, genes originating from landraces or modern cultivars can be more easily introgressed into new cultivars. There are more than 14,000 wheat landraces in the national seed bank of China, and landraces exhibiting high resistance to $B g t$ isolates have been identified in recent years; some of them confer broad-spectrum powdery mildew resistance, such as Niaomai (carrying Pm2c) (Xu et al. 2015), Chiyacao (carrying Pm24) (Huang and Röder 2011), Baihulu (carrying Pm24b) (Xue et al. 2012), and Hulutou (carrying MlHLT) (Wang et al. 2015). Datoumai (DTM), a Chinese wheat landrace from the national seed bank, also showed high resistance to more than 20 prevalent $B g t$ isolates in China. In this study, we report: (i) characterization of the DTM powdery mildew resistance gene PmDTM; (ii) fine mapping and identification of the candidate genes of PmDTM; and (iii) development of breeder-friendly polymerase chain reaction (PCR)-based markers and kompetitive allele-specific PCR (KASP) markers for marker-assisted introgression of PmDTM into modern elite lines.

\section{Materials and Methods}

Plant materials and pathogen isolates. DTM was crossed with Huixianhong (HXH), which is highly susceptible to powdery mildew. Twenty-three Chinese Bgt isolates were used to compare the reactions of DTM, HXH, and 11 wheat accessions with known powdery mildew resistance genes (Table 1). Isolate E09 was used to inoculate parental lines DTM and $\mathrm{HXH}$ and the $\mathrm{F}_{1}, \mathrm{~F}_{2}$, and $\mathrm{F}_{2: 3}$ progenies derived from the cross DTM $\times \mathrm{HXH}$. A total of $669 \mathrm{~F}_{2}$ plants from the cross Chiyacao $(P m 24) \times$ DTM were employed to analyze the genetic relationship between PmDTM and Pm24. Furthermore, 576 core wheat germplasm accessions from the Chinese Academy of Agricultural Sciences were used for frequency analysis of the PmDTM candidate gene.

Powdery mildew evaluations. A protocol described by $\mathrm{Xu}$ et al. (2015) was used to evaluate powdery mildew response. In brief, the $\mathrm{F}_{2}$ populations and 20 to 25 seedlings from each $\mathrm{F}_{2: 3}$ family were evaluated for responses to $B g t$ isolate E09 in a growth chamber. Each $\mathrm{F}_{2}$ plant was planted in a single cell $(3 \times 3 \mathrm{~cm})$ of a 72 -cell tray, and DTM and HXH were used as resistant and susceptible checks, respectively. All seedlings at the two-leaf stage were inoculated with fresh conidiospores and incubated in a chamber with a daily cycle of $16 \mathrm{~h}$ of light at $22^{\circ} \mathrm{C}$ and $8 \mathrm{~h}$ of darkness at $18^{\circ} \mathrm{C}$. The infection type (IT) was recorded 7 to 10 days postinoculation when HXH displayed severe symptoms and signed with a 0 to 4 scale; $0=$ no visible symptoms and signs; $0 ;=$ necrotic flecks without sporulation; 1 = sparse aerial hypha and little sporulation, diameter of colonies less than $1 \mathrm{~mm} ; 2$ = moderate aerial hyphae and sporulation, diameter of colonies less than $1 \mathrm{~mm} ; 3$ = thick aerial hypha and abundant sporulation, diameter of colonies more than $1 \mathrm{~mm}$; and $4=$ abundant sporulation with more than $80 \%$ of the leaf area covered with aerial hyphae. The values of 0 to 2 were classified as resistant, and those of 3 to 4 were classified as susceptible (Xu et al. 2015).

Preliminary and fine mapping of PmDTM. Genomic DNA was extracted from young leaves according to the method of Zhao et al. (2019). PCR was performed following the procedure of Zhang et al. (2017). The PCR products were separated in either $8 \%$ nondenaturing polyacrylamide gels (Acrylamide:Bisacrylamide $=19: 1$ or 29:1) or $1 \%$ agarose gels.

DNA bulks were prepared for bulked segregant analysis by pooling equal amounts of DNA from 10 homozygous resistant $F_{2}$ plants or 10 homozygous susceptible plants. Together with DNA from the two parents, the resistant and susceptible bulks were genotyped with the Wheat 55K SNP Array (Zhang et al. 2019). Finally, a 42.8-Mb interval on chromosome 1DS was found to be associated with the powdery mildew resistance gene in DTM based on the Chinese Spring (CS) reference sequence IWGSC RefSeq version 1.0 (IWGSC 2018). Linkage analysis with $200 \mathrm{~F}_{2}$ plants and four known simple sequence repeat (SSR) markers located on chromosome 1DS delimited PmDTM to the interval between Xgwm337 and Xbarc229. To fine map PmDTM, more recombinants were identified from a large $\mathrm{F}_{2}$ population by genotyping with these two flanking markers. The CS reference sequence corresponding to the target interval was used to screen for new SSR markers with the Perl script MISA program (https://webblast.ipk-gatersleben.de/misa/). Three highly confident genes (TraesCS1D02G058800, TraesCS1D02G058900, and TraesCS1D02G059000) residing within the PmDTM region were amplified and sequenced to find single nucleotide polymorphisms (SNPs) and insertion-deletion polymorphisms (InDel) between DTM and HXH, which were used to develop KASP or PCR-based markers. All primers were designed with MacVector 11.0 (Accelrys, Cambridge, U.K.). Primer information of the markers detecting polymorphisms is shown in Supplementary Table 1.

KASP genotyping assays. KASP assays were performed on a LightCycler 480 II system (Roche, Indianapolis, IN). Reaction mixtures consisted of final volumes of $10 \mu$ l containing $5 \mu$ l of $2 \times$ KASP Master mix (LGC Genomics), $0.14 \mu$ l of primer mix (12 $\mu \mathrm{M}$ of each allele-specific primer and $30 \mu \mathrm{M}$ of common primer), and $5 \mu \mathrm{l}$

Table 1. Differential reactions of resistant Datoumai (DTM), susceptible Huixianhong (HXH), and 11 accessions with known powdery mildew resistance genes to 23 isolates of Blumeria graminis f. sp. tritici (Bgt) collected from different regions of China

\begin{tabular}{|c|c|c|c|c|c|c|c|c|c|c|c|c|c|c|c|c|c|c|c|c|c|c|c|c|}
\hline \multirow[b]{2}{*}{ Cultivar/line } & \multirow[b]{2}{*}{$P m$} & \multicolumn{23}{|c|}{ Bgt isolate } \\
\hline & & $\overline{\text { B13 }}$ & B14 & B15 & B17 & B18 & B41 & B48 & B49 & B50 & B56 & E05 & E06 & E07 & E09 & E17 & E18 & E20 & E21 & E23 & E31 & E32 & E49 & $\overline{\text { E50 }}$ \\
\hline $\begin{array}{l}\text { Axminster/ } \\
8^{*} \mathrm{Cc}^{\mathrm{a}}\end{array}$ & $P m 1$ & 4 & 0 & 4 & 4 & 4 & 4 & 4 & 4 & 4 & 4 & 3 & 4 & 4 & 4 & 4 & 4 & 4 & 4 & 4 & 4 & 3 & 4 & $\overline{4}$ \\
\hline $\mathrm{Ulka} / 8 * \mathrm{Cc}$ & Pm2 & 0 & 4 & 0 & 0 & 4 & 0 & 0 & 0 & 4 & 0 & 0 & 0 & 0 & 0 & 0 & 4 & 0 & 4 & 0 & 0 & 0 & 0 & 0 \\
\hline Khapli// $8 * \mathrm{Cc}$ & Pm $4 a$ & 0 & 0 & 0 & 0 & 4 & 1 & 0 & 0 & 4 & 4 & 0 & 1 & 0 & 0 & 4 & 4 & 0 & 4 & 0 & 4 & 0 & 0 & 0 \\
\hline Armada & Pm $4 b$ & 0 & 0 & 0 & 0 & 4 & 0 & 0 & 0 & 4 & 4 & 0 & 0 & 0 & 0 & 4 & 4 & 0 & 4 & 0 & 4 & 0 & 0 & 1 \\
\hline Fuzhuang 30 & Pm5e & 0 & 1 & 4 & 3 & 0 & 4 & 0 & 3 & 0 & 0 & 0 & 4 & 0 & 0 & 1 & 0 & 3 & 0 & 3 & 0 & 0 & 4 & 0 \\
\hline Timgalen & Pm6 & 4 & 4 & 4 & 3 & 4 & 4 & 4 & 4 & 4 & 4 & 4 & 4 & 4 & 4 & 4 & 4 & 4 & 4 & 4 & 4 & 4 & 4 & 4 \\
\hline $\begin{array}{c}\text { Yangmai5/ } \\
\text { Sub.6V }\end{array}$ & $\operatorname{Pm} 21$ & 0 & 0 & 0 & 0 & 0 & 0 & 0 & 0 & 0 & 0 & 0 & 0 & 0 & 0 & 0 & 0 & 0 & 0 & 0 & 0 & 0 & 0 & 0 \\
\hline $81-7241$ & $P m 23$ & 0 & 0 & 0 & 0 & 4 & 0 & 0 & 0 & 4 & 4 & 0 & 0 & 0 & 0 & 4 & 4 & 0 & 0 & 0 & 4 & 0 & 0 & 0 \\
\hline Chiyacao & Pm24 & 0 & 0 & 0 & 0 & 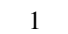 & 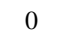 & 0 & 0 & 0 & 0 & 3 & 1 & 0 & 0 & 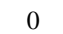 & 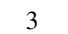 & 0 & 3 & 0 & 3 & 0 & 0 & 0 \\
\hline Hulutou & MlHLT & 0 & 0 & 3 & 1 & 0 & 0 & 1 & 0 & 0 & 0 & 0 & 0 & 0 & 0 & 0 & 3 & 1 & 0 & 0 & 1 & 0 & 0 & 1 \\
\hline $5 \mathrm{P} 27$ & Pm 30 & 0 & 4 & 0 & 0 & 4 & 0 & 0 & 0 & 4 & 0 & 0 & 0 & 1 & 0 & 0 & 4 & 0 & 3 & 0 & 0 & 0 & 0 & 0 \\
\hline DTM & PmDTM & 0 & 0 & 0 & 0 & 0 & 0 & 0 & 0 & 0 & 0 & 0 & 0 & 0 & 0 & 1 & 3 & 0 & 0 & 0 & 1 & 0 & 0 & 0 \\
\hline HXH & $\ldots$ & 4 & 4 & 4 & 4 & 4 & 4 & 4 & 4 & 4 & 4 & 4 & 4 & 4 & 4 & 4 & 4 & 4 & 4 & 4 & 4 & 4 & 4 & 4 \\
\hline
\end{tabular}

a Seven times backcrossed to cultivar Chancellor. 
(100 ng) of genomic DNA. The cycling conditions were as follows: $94^{\circ} \mathrm{C}$ for $15 \mathrm{~min}, 10$ cycles of $94^{\circ} \mathrm{C}$ for $20 \mathrm{~s}$, touchdown starting at $61^{\circ} \mathrm{C}$ for $60 \mathrm{~s}$ (decreasing $0.6^{\circ} \mathrm{C}$ per cycle), 32 cycles of $94^{\circ} \mathrm{C}$ for $20 \mathrm{~s}, 55^{\circ} \mathrm{C}$ for $60 \mathrm{~s}$, and a final KASP reading step of $37^{\circ} \mathrm{C}$ for $60 \mathrm{~s}$. The end-point fluorescence data were visualized and analyzed by LightCycler 480 software version 1.5.1.62.

Isolation and sequencing of the full-length cDNA of PmDTM candidate gene. Total RNA was extracted with TRIzol reagent (Invitrogen) according to the method of $\mathrm{Su}$ et al. (2019). First-strand cDNA was synthesized with HiScript II (Vazyme, Nanjing, China) using Oligo $(\mathrm{dT})_{23}$ primer. Nested PCR was carried out first with STK_UTR_F1 and STK_UTR_R1, and then with STK_UTR_F2 and STK_UTR_R2 (Supplementary Table 1). The PCR products were purified, cloned, and sequenced.

Quantitative reverse transcription PCR (qRT-PCR) analysis. For gene expression assays, 7-day-old seedlings of DTM and HXH were treated with $B g t$ isolate E09. Samples from different plants were collected immediately before inoculation $(0 \mathrm{~h})$ and at $3,6,12,24,48$, and $72 \mathrm{~h}$ postinoculation (hpi). The cDNAs were obtained from $2 \mu \mathrm{g}$ of total RNA using a reverse transcription kit HiScript Q RT SuperMix for qPCR (Vazyme). qRT-PCR reactions were performed with primer STK-E5 and AceQ Universal SYBR qPCR Master Mix (Vazyme) following the previously described procedure (Long et al. 2018). The TaGAPDH gene was used as an internal control, and the $2^{-\Delta \Delta \mathrm{Ct}}$ method was used to calculate the relative gene expression (Livak and Schmittgen 2001). All primers for qRT-PCR assays are listed in Supplementary Table 1.

Frequency analysis of the resistant allele. The PCR-based markers STK-E5 and STK-CS representing the critical variation of the PmDTM candidate gene were used to determine the presence or absence of a resistant allele in 576 wheat accessions including 76 Chinese landraces, 353 Chinese modern cultivars, and 147 foreign cultivars. The primers of STK-E5 and STK-CS were designed according to the genomic sequences of DTM and CS, respectively. Their forward primer sequences are highly variable (Supplementary Table 1).

Data analyses. $\chi^{2}$ tests were performed to test the hypothesis that a dominant powdery mildew resistance allele conferred resistance in the $F_{2}$ and $F_{2: 3}$ populations. The genetic linkage map (Supplementary Fig. 2) was calculated using the maximum likelihood algorithm and the Kosambi function in JoinMap 4.0 (Van Ooijen 2006). The translated protein sequences of the PmDTM candidate gene were used as queries to search the NCBI Conserved Domains Database for domain analysis (https://www.ncbi.nlm.nih.gov/Structure/cdd/docs/cdd_ search.html).

\section{Results}

Inheritance of the powdery mildew resistance in DTM. Segregating populations from DTM $\times \mathrm{HXH}$ and the parents were tested with $B g t$ isolate E09 at the seedling stage. DTM was immune with

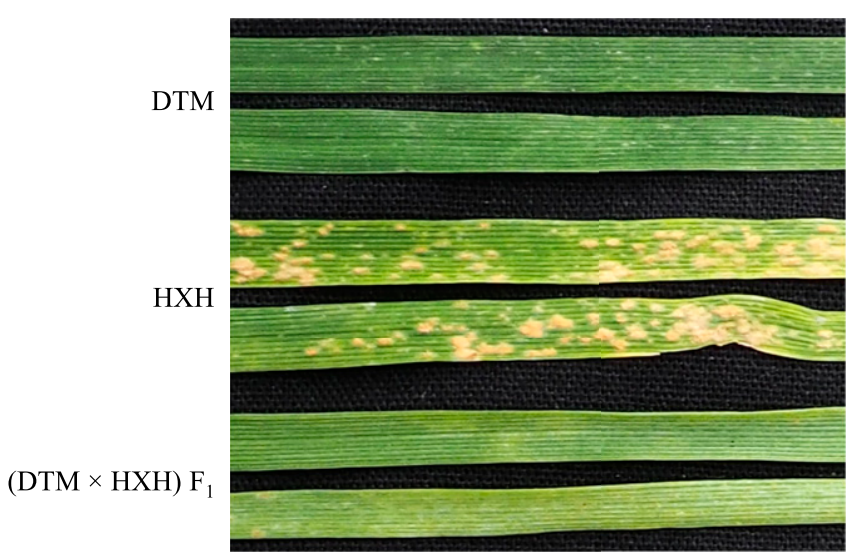

Fig. 1. Powdery mildew reactions of resistant parent Datoumai (DTM), susceptible parent Huixianhong $(\mathrm{HXH})$, and their $\mathrm{F}_{1}$ hybrids to Blumeria graminis $\mathrm{f}$. sp. tritici isolate E09.
IT 0 , and $\mathrm{HXH}$ was highly susceptible with IT 4 (Fig. 1). The $\mathrm{F}_{1}$ hybrids were highly resistant (IT 0) to E09, indicating the dominant nature of the powdery mildew resistance in DTM. Among $200 \mathrm{~F}_{2}$ plants, 156 were resistant (IT 0 to 1 ) and 44 susceptible (IT 3 to 4 ), fitting a $3: 1$ ratio $\left(\chi^{2}=0.96, P>0.05\right)$. The $\mathrm{F}_{2: 3}$ populations segregated as 50 homozygous resistant, 106 segregating, and 44 homozygous susceptible, fitting a 1:2:1 segregation ratio $\left(\chi^{2}=1.08, P>\right.$ $0.05)$. These results suggested that the powdery mildew resistance in DTM was controlled by a single dominant gene, temporarily designated as PmDTM.

Preliminary mapping of $P m D T M$ and its relationship with Pm24. To localize PmDTM in the wheat genome, the parental lines as well as the resistant and susceptible DNA bulks were genotyped with the Wheat 55K SNP Array. Out of 553 polymorphic SNPs identified between the parental lines as well as the DNA bulks, 136 SNPs were distributed on chromosome 1D (Supplementary Fig. 1). Further analysis indicated that 101 SNPs were clustered in a 42.8$\mathrm{Mb}$ genomic region (7.4 to $50.2 \mathrm{Mb}$ ) on chromosome 1DS. The known SSR markers located on 1DS were subsequently surveyed for polymorphisms. Linkage mapping with $200 \mathrm{~F}_{2}$ plants and four polymorphic markers (Xgwm337, Xbarc229, Xgpw4503, and $X c f d 83)$ initially localized PmDTM into a genetic interval flanked by Xgwm337 and Xbarc229 (Fig. 2A). A comparison with published genetic maps suggested that PmDTM was located in the same chromosomal region as Pm24 (Supplementary Fig. 2; Huang and Röder 2011), Pm24b (Xue et al. 2012), and MlHLT (Wang et al. 2015). The $\mathrm{F}_{2}$ population consisting of 669 plants derived from Chiyacao $(P m 24) \times$ DTM was inoculated with isolate E09, and no visible symptom was observed for all $\mathrm{F}_{2}$ plants. On the basis of these results, we proposed that the resistance gene in DTM was mapped to the same locus as $P m 24$.

Powdery mildew reactions of DTM and accessions with known genes. Differential reactions of DTM, HXH, and 11 wheat accessions possessing known powdery mildew resistance genes to $23 \mathrm{Bgt}$ isolates are listed in Table 1. Pm6 has lost resistance to all Chinese Bgt isolates used in this study. DTM was highly resistant to 22 isolates and was susceptible only to isolate E18 (Table 1). Two landraces, Chiyacao and Hulutou, also conferred broad-spectrum

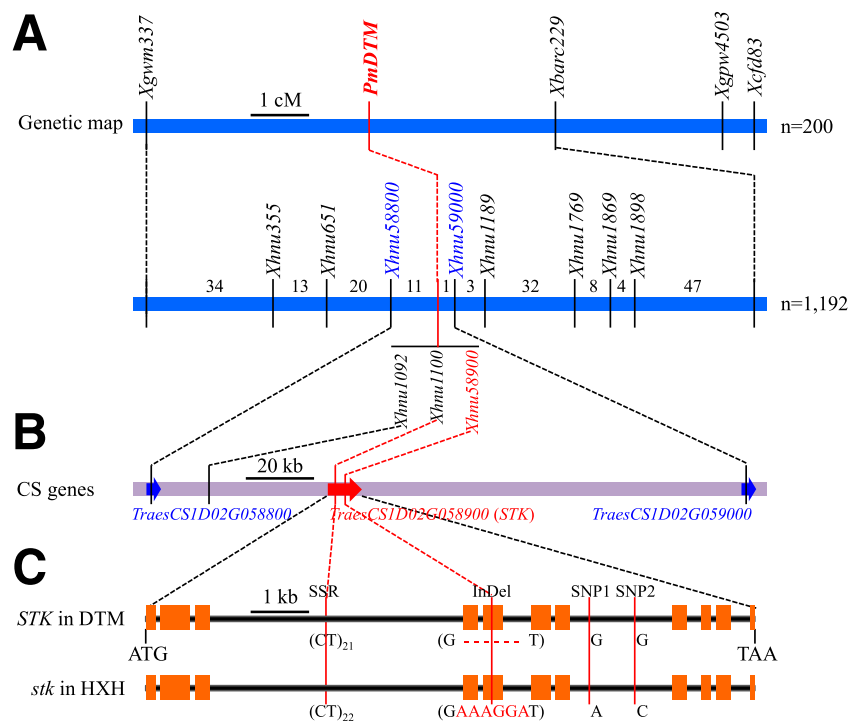

Fig. 2. Map-based cloning of PmDTM. A, High-density genetic map of the 1DS region containing PmDTM. The maps are constructed based on 200 (upper) and 1,192 (lower) $\mathrm{F}_{2}$ plants. Numbers of crossovers identified between the adjacent marker loci are shown above the rectangle. B, Genes annotated within the $180.5-\mathrm{Kb}$ candidate region according to the IWGSC CS RefSeq version 1.1 annotation. C, Gene structure of STK in resistant Datoumai (DTM) and susceptible Huixianhong $(\mathrm{HXH})$. Orange rectangles represent exons, and black lines represent introns. Four lettered vertical lines represent variations of STK between DTM and HXH, including one SSR, one 6-bp InDel, and two SNPs. 
resistance to powdery mildew but were susceptible to four and two $B g t$ isolates, respectively (Table 1). The landrace Baihulu was not included in this study due to being unavailable in our laboratory. However, Baihulu has been reported to be resistant to isolate E18 (Xue et al. 2012). Thus, the PmDTM gene in DTM gave a unique reaction pattern that was different from other resistance alleles at the Pm24 locus.

Fine mapping of PmDTM. For the saturation of the genetic linkage map of the PmDTM region, a total of eight polymorphic SSR markers were developed based on the 29.6-Mb CS reference sequence corresponding to the Xgwm337 to Xbarc229 interval (Supplementary Figs. 2 and 3). To fine map PmDTM, 168 recombinants harboring 173 crossovers were identified between the flanking markers Xgwm337 and Xbarc229 from 1,192 DTM $\times \mathrm{HXH} \mathrm{F}_{2}$ plants (Fig. 2). These recombinants were genotyped with the newly developed SSR markers and phenotyped with their $\mathrm{F}_{2: 3}$ progenies. Further linkage analysis indicated that PmDTM was flanked by Xhnu651 and Xhnu1189, and cosegregated with Xhnu1092 and Xhnul100 (Fig. 2A; Supplementary Fig. 2). The SSR marker Xhnu1092 was located in an intergenic region, whereas $X h n u 1100$ was located within gene TraesCS1D02G058900 (Fig. 2B) according to the IWGSC CS RefSeq version 1.1 annotation (https://urgi.versailles.inra.fr/download/ iwgsc/IWGSC_RefSeq_Annotations/v1.1/). We sequenced and compared the full-length genomic sequences of TraesCS1D02G058900 and adjacent TraesCS1D02G058800 and TraesCS1D02G059000. A total of six variations were identified between DTM and HXH. There was only one C/T SNP that occurred in TraesCS1D02G058800 and only one T/C SNP that occurred in TraesCS1D02G059000. These two nonsynonymous SNPs were converted to KASP makers (Fig. 3A and C) and were used to genotype the 168 recombinants. We identified 11 crossovers between Xhnu58800 (TraesCS1D02G058800) and PmDTM, and only one crossover between PmDTM and Xhnu59000 (TraesCS1D02G059000) (Fig. 2A). Except for the SSR marker
Xhnu1100 mentioned above, three additional variations in TraesCS1D02G058900 were identified between DTM and HXH, including a 6-bp InDel and two SNPs. The 6-bp InDel is located within the predicted exon 5 (Fig. 2C). We developed a KASP marker (Xhnu58900, Fig. 3B) and a breeder-friendly PCR marker (STK-E5, Fig. 3D) based on the 6-bp variation to genotype all the recombinant plants. As expected, this InDel variation (Xhnu58900) was cosegregated with PmDTM (Fig. 2A). Because only one recombinant (P5-236) was identified between Xhnu58900 and Xhnu59000, we further checked the genotypes and phenotypes of $43 \mathrm{~F}_{3}$ plants derived from P5-236 (Supplementary Table 2). All the $F_{3}$ plants were resistant to $B g t \mathrm{E} 09$, which was in coincidence with the genotypes checked by Xhnu58900. Thus, PmDTM was finally fine mapped to a $0.5-\mathrm{cM} X h n u 58800$ to Xhnu59000 interval covering $180.5 \mathrm{~Kb}(38,728,125$ to $38,908,656 \mathrm{bp})$ on chromosome 1DS, and TraesCS1D02G058900 was the most likely candidate gene for PmDTM (Fig. 2; Supplementary Fig. 2).

Candidate gene analysis. We compared a full-length cDNA of TraesCS1D02G058900 with the corresponding genomic sequence and determined that this gene consists of 11 exons and 10 introns (Fig. 2C). TraesCS1D02G058900 in DTM spans 10,410 bp from the starting ATG to the termination TAA, with a complete coding sequence of 2,682 bp (Supplementary Fig. 4). TraesCS1D02G058900 encodes a receptor-like protein with two putative serine/threonine kinase (STK) domains in tandem (Fig. 4) and is designated here as STK. Comparison of the complete STK region in DTM revealed a deletion of two amino acids (K [Lys] and G [Gly] at positions 400 and 401) relative to the susceptible parent HXH (Fig. 4). The KG deletion resides in the STK1 domain and may be critical for the resistance to powdery mildew.

Transcript levels of $S T K$ relative to $T a G A P D H$ were analyzed in DTM and HXH by qRT-PCR (Fig. 5). The basal transcript levels of $S T K$ in DTM were 1.5-fold higher than in $\mathrm{HXH}$ immediately

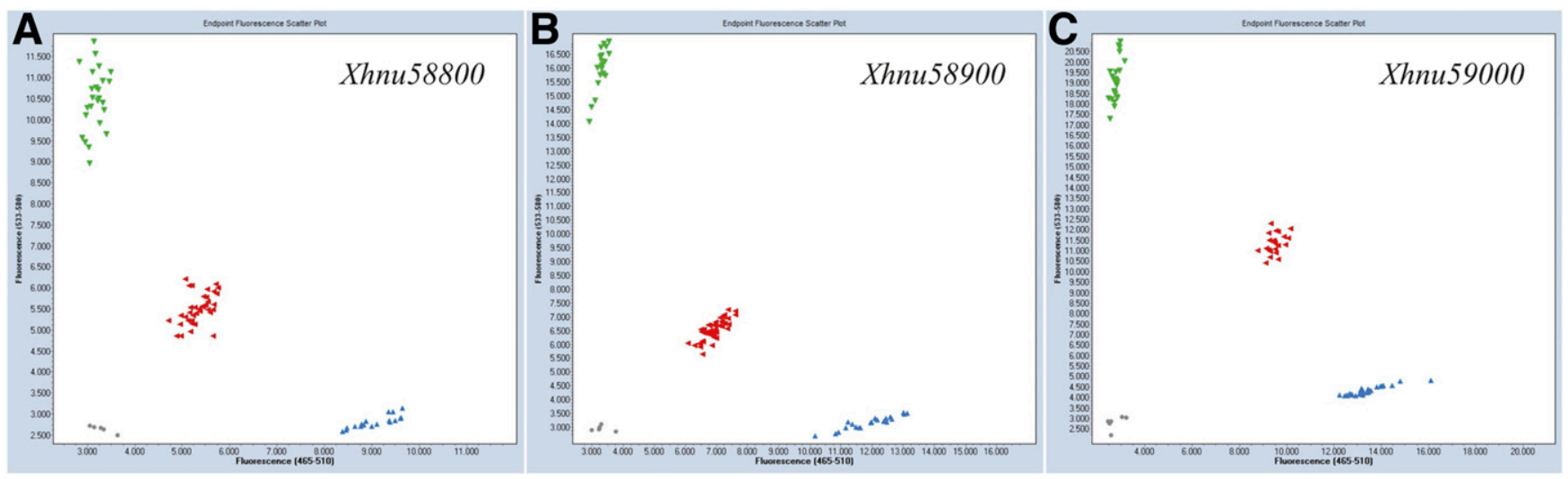

D

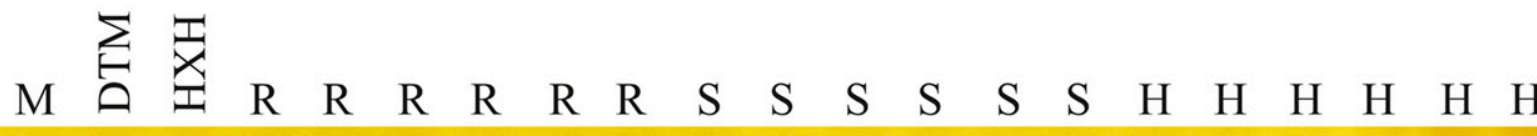

$100 \mathrm{bp}$

Fig. 3. KASP and PCR-based markers developed in this study. The KASP markers are developed according to the SNP in TraesCS1D02G058800 (A), InDel in TraesCS1D02G058900 (B), and SNP in TraesCS1D02G059000 (C). The blue (bottom right) and green (upper left) triangles represent resistant Datoumai (DTM) and susceptible Huixianhong $(\mathrm{HXH}$ ) alleles, respectively. The red (center) triangles represent heterozygous alleles. The gray dots (bottom left) represent the negative control. PCR marker STK-E5 (D) was developed according to the 6-bp variation in exon 5 of TraesCS1D02G058900. R, S, and H indicate resistant, susceptible, and heterozygous plants, respectively, in the $F_{2}$ population. $M$ is the DNA size standard. 
before the time of inoculation $(0 \mathrm{~h})$. The expression of STK in DTM was rapidly down-regulated by the presence of the pathogen and reached the lowest level at $12 \mathrm{hpi}$. The $S T K$ expression pattern in HXH was similar to that in DTM, although different expression levels were detected between them throughout the time course except 24 hpi (Fig. 5). These results suggested that $S T K$ was transiently induced by the infection of $B g t \mathrm{E} 09$, and the relative abundance of the transcript varied and differed between the resistant and susceptible parents.

Frequency analysis of the $S T K$ allele with 6-bp deletion. The PCR-based markers STK-E5 and STK-CS developed on the basis of the 6-bp variation were used to analyze the frequency of the resistant $S T K$ allele in 576 wheat accessions. Single bands were amplified in 429 accessions with $S T K-E 5$, and the rest were successively amplified with $S T K-C S$ (data not shown). Surprisingly, the allele with 6-bp deletion was only present in three Chinese landraces: DTM, Chiyacao, and Hulutou. All of the 353 Chinese modern cultivars and 147 foreign cultivars carry the susceptible stk allele, implying that there is a great potential to utilize the rare allele with 6-bp deletion for further genetic improvement. To confirm this observation, we cloned the full-length cDNA of STK from Chiyacao (Pm24), Hulutou $(M l H L T)$, and Fielder. As shown in Supplementary Figure 4, Chiyacao and Hulutou have the same coding sequence as DTM, and Fielder (an excellent receptor for wheat genetic transformation) is susceptible to $B g t$ and has the same coding sequence as $\mathrm{HXH}$. These results further demonstrate that PmDTM, Pm24, and MlHLT are mapped to the same locus, and $S T K$ is the most likely candidate gene of them.

\section{Discussion}

Wheat landraces are abundant in China and show wide genetic diversity for powdery mildew resistance. Up to now, more than 10 powdery mildew resistance genes or alleles have been identified from Chinese wheat landraces (Tan et al. 2019). In the present study, a broad-spectrum powdery mildew resistance gene, PmDTM, in Chinese landrace DTM was fine mapped using a large population with $1,192 \mathrm{~F}_{2}$ plants. Based on the genotypes at the gene interval and phenotypes of the identified recombinants, PmDTM was placed in the 0.5-cM Xhnu58800 to Xhnu59000 interval on 1DS. Chromosome 1DS also harbors three known powdery mildew resistance genes, including Pm24 (Huang and Röder 2011), Pm24b (Xue et al. 2012), and MlHLT (Wang et al. 2015). Fine mapping and map-based cloning of these disease resistance genes helps to clarify the exact genetic relationship among them.

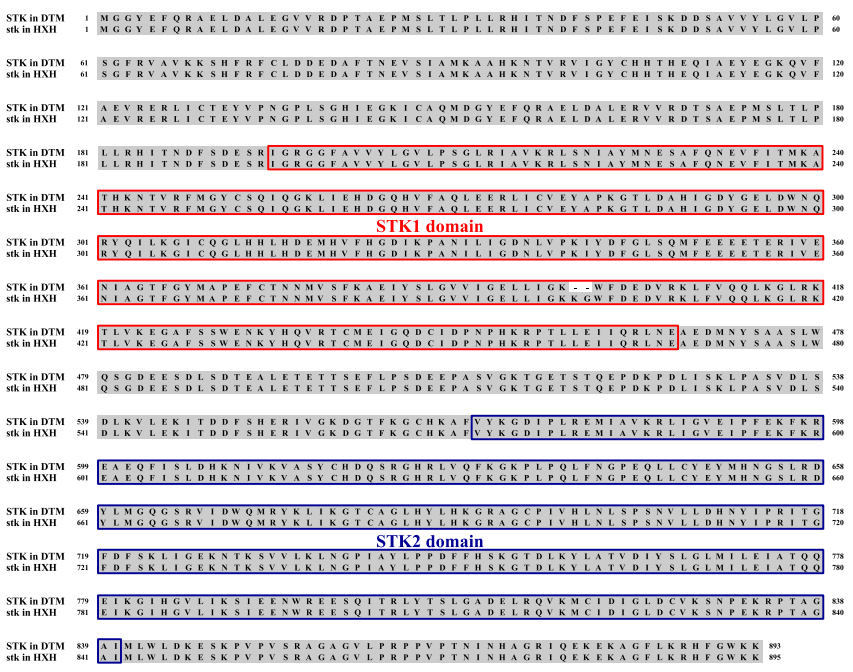

Fig. 4. Protein sequence alignment between STK in resistant Datoumai (DTM) and stk in susceptible Huixianhong (HXH). Domains in the protein sequences were predicted based on the NCBI Conserved Domains Database. Two putative serine/threonine kinase (STK) domains were separately marked with red and blue rectangles.
PmDTM was finally mapped to the region between TraesCS1D02G058800 and TraesCS1D02G059000, in a 180,531 bp genomic region based on the CS genome sequence (Fig. 2C). Only one highly confident gene, TraesCS1D02G058900 (STK), was annotated within this region (Fig. 2B). STK encodes a receptor-like protein kinase with 893 and 895 amino acids in DTM and HXH, respectively (Fig. 4). The KASP marker Xhnu58900 developed according to the 6-bp variation in STK always cosegregated with the phenotypes. Thus, we concluded that $S T K$ was the only reasonable candidate gene for PmDTM, and further genetic transformation experiments are needed to validate its role in resistance to Bgt E09.

To prevent the infection of pathogen, plants have evolved distinct resistance mechanisms including effector-triggered immunity, PAMPtriggered immunity, and quantitative disease resistance (Krattinger and Keller 2016). Most of the powdery mildew resistance genes cloned so far confer race-specific resistance, such as Pm2, Pm3, Pm8, Pm21, and Pm60 (He et al. 2018; Hurni et al. 2013; SánchezMartin et al. 2016; Yahiaoui et al. 2004; Zou et al. 2018). They encode NB-LRR-type proteins and activate effector-triggered immunity. By contrast, STK encodes a protein containing two putative STK domains (Fig. 4). To date, only two wheat disease resistance genes, $\operatorname{Yr} 15$ (Klymiuk et al. 2018) and Sr60 (Chen et al. 2020), were found to possess two tandem kinase or pseudokinase domains. The expression pattern of STK was similar to $\mathrm{Yr} 15$; both were downregulated by the presence of the pathogen (Fig. 5). The deletion of two amino acids (KG) was located within the STK1 domain, and the STK2 domain was identical between DTM and HXH (Fig. 4). For this phenomenon, we speculated that the KG deletion might alter the kinase activity and eventually lead to the different response of the host to $B g t$ isolates.

Cloning and sequencing of STK in DTM, Chiyacao, and Hulutou indicated that PmDTM was the same as previously designated $P m 24$ and $M l H L T$, although they showed some differences in reaction to 23 Chinese Bgt isolates (Table 1). The different resistance spectrum may be mainly caused by the variations in genetic background. The same resistance genes but with a different spectrum also occurred at the Pm2 locus (Chen et al. 2019).

Rare genetic variants are abundant in plants and are expected to contribute to disease resistance and other important agronomical traits. For example, scab resistance gene $F h b 1$ was only present in a few Chinese accessions such as Wangshuibai and Sumai 3 (Li et al. 2019b); the GS2 allele with enlarged grain size and enhanced grain yield came from a rare mutation of the microRNA target site (Hu et al. 2015); the most favorable haplotypes of $\operatorname{crtRB1}$, encoding $\beta$-carotene hydroxylase 1 , were detected only in about $2 \%$ of the maize germplasms (Yan et al. 2010). In the present study, the resistant STK allele with a 6-bp deletion only existed in three Chinese landraces out of 576 wheat accessions. These results suggested that

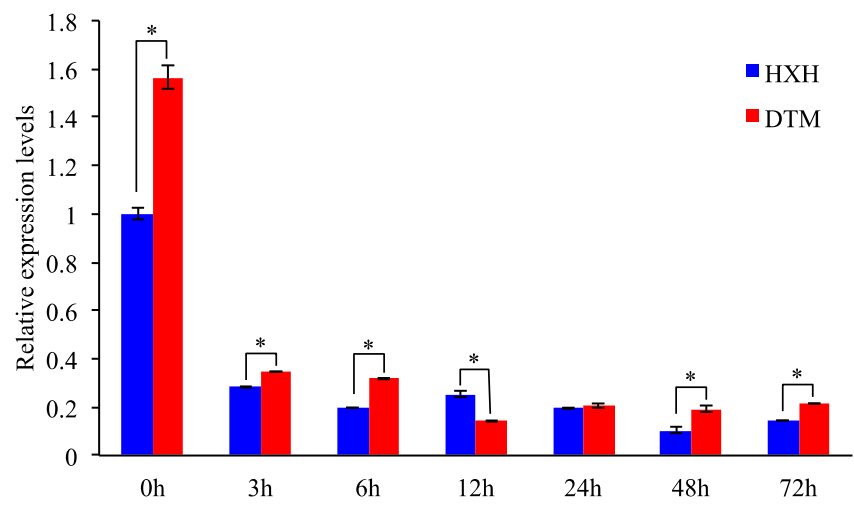

Fig. 5. Transcript levels of STK in Blumeria graminis f. sp. tritici-inoculated Huixianhong $(\mathrm{HXH})$ and Datoumai (DTM) plants. Samples were collected immediately before inoculation $(0 \mathrm{~h})$ and at $3,6,12,24,48$, and $72 \mathrm{~h}$ postinoculation. The expression levels were normalized against the internal control gene TaGAPDH. Error bars denote $\pm \mathrm{SD}(n=3)$. Asterisk $\left({ }^{*}\right)$ indicates $P<0.01$ (Student's $t$ test). 
the rare allele with a 6-bp deletion has not yet been artificially selected and thus could be widely exploited for wheat resistance improvement. Based on the 6-bp variation in $S T K$, we have developed a codominant, breeder-friendly PCR-based marker $S T K$ E5 for marker-assisted selection and a KASP marker Xhnu58900 for high-throughput detection of PmDTM (Fig. 3B and D). With these two functional markers, we are introgressing PmDTM into commercially successful cultivars by implementing both forward selection and background selection. Breeders can use these developed near-isogenic lines for PmDTM to produce new powdery mildew resistant cultivars in the near future.

\section{Acknowledgments}

We thank J. Jia of the Chinese Academy of Agricultural Sciences for providing the core wheat germplasm accessions for this study. We also thank J. Sun of the Chinese Academy of Agricultural Sciences for inspiring discussions and support.

\section{Literature Cited}

Chen, F., Jia, H. Y., Zhang, X. J., Qiao, L. Y., Li, X., Zheng, J., Guo, H. J., Powers, C., Yan, L. L., and Chang, Z. J. 2019. Positional cloning of PmCH1357 reveals the origin and allelic variation of the $P m 2$ gene for powdery mildew resistance in wheat. Crop J. 7:771-783.

Chen, S., Rouse, M. N., Zhang, W., Zhang, X., Guo, Y., Briggs, J., and Dubcovsky, J. 2020. Wheat gene Sr60 encodes a protein with two putative kinase domains that confers resistance to stem rust. New Phytol. 225:948-959.

Chen, X. M., Luo, Y. H., Xia, X. C., Xia, L. Q., Chen, X., Ren, Z. L., He, Z. H., and Jia, J. Z. 2005. Chromosomal location of powdery mildew resistance gene Pm16 in wheat using SSR marker analysis. Plant Breed. 124:225-228.

Conner, R. L., Kuzyk, A. D., and Su, H. 2003. Impact of powdery mildew on the yield of soft white spring wheat cultivars. Can. J. Plant Sci. 83:725-728.

Friebe, B., Heun, M., Tuleen, N., Zeller, F. J., and Gill, B. S. 1994. Cytogenetically monitored transfer of powdery mildew resistance from rye into wheat. Crop Sci. 34:621-625.

He, H., Zhu, S., Zhao, R., Jiang, Z., Ji, Y., Ji, J., Qiu, D., Li, H., and Bie, T. 2018. Pm21, encoding a typical CC-NBS-LRR protein, confers broad-spectrum resistance to wheat powdery mildew disease. Mol. Plant 11:879-882.

Hu, J., Wang, Y., Fang, Y., Zeng, L., Xu, J., Yu, H., Shi, Z., Pan, J., Zhang, D., Kang, S., Zhu, L., Dong, G., Guo, L., Zeng, D., Zhang, G., Xie, L., Xiong, G., Li, J., and Qian, Q. 2015. A rare allele of GS2 enhances grain size and grain yield in rice. Mol. Plant 8:1455-1465.

Huang, X. Q., and Röder, M. S. 2004. Molecular mapping of powdery mildew resistance genes in wheat: A review. Euphytica 137:203-223.

Huang, X. Q., and Röder, M. S. 2011. High-density genetic and physical bin mapping of wheat chromosome 1D reveals that the powdery mildew resistance gene $P m 24$ is located in a highly recombinogenic region. Genetica 139:1179-1187.

Hurni, S., Brunner, S., Buchmann, G., Herren, G., Jordan, T., Krukowski, P., Wicker, T., Yahiaoui, N., Mago, R., and Keller, B. 2013. Rye Pm8 and wheat $P m 3$ are orthologous genes and show evolutionary conservation of resistance function against powdery mildew. Plant J. 76:957-969.

International Wheat Genome Sequencing Consortium (IWGSC). 2018. Shifting the limits in wheat research and breeding using a fully annotated reference genome. Science 361:661.

Jia, J., Devos, K. M., Chao, S., Miller, T. E., Reader, S. M., and Gale, M. D. 1996. RFLP-based maps of the homoeologous group- 6 chromosomes of wheat and their application in the tagging of $P m 12$, a powdery mildew resistance gene transferred from Aegilops speltoides to wheat. Theor. Appl. Genet. 92:559-565.

Jones, J. D., and Dangl, J. L. 2006. The plant immune system. Nature 444:323-329.

Klymiuk, V., Yaniv, E., Huang, L., Raats, D., Fatiukha, A., Chen, S., Feng, L., Frenkel, Z., Krugman, T., and Lidzbarsky, G. 2018. Cloning of the wheat Yr15 resistance gene sheds light on the plant tandem kinase-pseudokinase family. Nat. Commun. 9:3735.

Krattinger, S. G., and Keller, B. 2016. Molecular genetics and evolution of disease resistance in cereals. New Phytol. 212:320-332.

Krattinger, S. G., Lagudah, E. S., Spielmeyer, W., Singh, R. P., Huerta-Espino, J., McFadden, H., Bossolini, E., Selter, L. L., and Keller, B. 2009. A putative ABC transporter confers durable resistance to multiple fungal pathogens in wheat. Science 323:1360-1363.

Li, G., Zhou, J., Jia, H., Gao, Z., Fan, M., Luo, Y., Zhao, P., Xue, S., Li, N., Yuan, Y., Ma, S., Kong, Z., Jia, L., An, X., Jiang, G., Liu, W., Cao, W., Zhang, R., Fan, J., Xu, X., Liu, Y., Kong, Q., Zheng, S., Wang, Y., Qin, B., Cao, S., Ding, Y., Shi, J., Yan, H., Wang, X., Ran, C., and Ma, Z. 2019b. Mutation of a histidine-rich calcium-binding-protein gene in wheat confers resistance to Fusarium head blight. Nat. Genet. 51:1106-1112.

Li, G. Q., Cowger, C., Wang, X. W., Carver, B. F., and Xu, X. X. 2019a. Characterization of Pm65, a new powdery mildew resistance gene on chromosome 2AL of a facultative wheat cultivar. Theor. Appl. Genet. 132: 2625-2632.

Liu, C., Cui, D., Zhao, J., Liu, N., Wang, B., Liu, J., Xu, E., Hu, Z., Ren, D., Tang, D., and Hu, Y. 2019. Two Arabidopsis receptor-like cytoplasmic kinases SZE1 and SZE2 associate with the ZAR1-ZED1 complex and are required for effector-triggered immunity. Mol. Plant 12:967-983.

Livak, K. J., and Schmittgen, T. D. 2001. Analysis of relative gene expression data using real-time quantitative PCR and the $2^{-\Delta \Delta \mathrm{Ct}}$ method. Methods 25:402-408.

Long, L., Guo, D. D., Gao, W., Yang, W. W., Hou, L. P., Ma, X. N., Miao, Y. C., Botella, J. R., and Song, C. P. 2018. Optimization of CRISPR/Cas9 genome editing in cotton by improved sgRNA expression. Plant Methods 14:85.

McIntosh, R. A., Dubcovsky, J., Rogers, W. J., Morris, C., and Xia, X. C. 2017 Catalogue of gene symbols for wheat. Suppl. Annu. Wheat Newsl. 53:1-20.

McIntosh, R. A., Yamazaki, Y., Dubcovsky, J., Rogers, J., Morris, C., Appels, R., and Xia, X. C. 2013. Catalogue of gene symbols for wheat. Pages 8-13 in: Proc. 12th Int. Wheat Genet. Symp., Yokohama, Japan. Y. Ogihara, ed.

Moore, J. W., Herrera-Foessel, S., Lan, C., Schnippenkoetter, W., Ayliffe, M., Huerta-Espino, J., Lillemo, M., Viccars, L., Milne, R., Periyannan, S., Kong, X., Spielmeyer, W., Talbot, M., Bariana, H., Patrick, J. W., Dodds, P., Singh, R., and Lagudah, E. 2015. A recently evolved hexose transporter variant confers resistance to multiple pathogens in wheat. Nat. Genet. 47:1494-1498.

Samobor, V., Vukobratović, M., and Jošt, M. 2006. Effect of powdery mildew attack on quality parameters and experimental bread baking of wheat. Acta Agric. Slov. 87:381-391.

Sánchez-Martín, J., Steuernagel, B., Ghosh, S., Herren, G., Hurni, S., Adamski, N., Vrána, J., Kubaláková, M., Krattinger, S. G., Wicker, T., Doležel, J., Keller, B. and Wulff, B. B. 2016. Rapid gene isolation in barley and wheat by mutant chromosome sequencing. Genome Biol. 17:221.

Singh, R. P., Singh, P. K., Rutkoski, J., Hodson, D. P., He, X., Jørgenssen, L. N., and Huertaespino, J. 2016. Disease impact on wheat yield potential and prospects of genetic control. Annu. Rev. Phytopathol. 54:303-322.

Su, Q., Yang, J., Fu, Q. Y., Jia, F. Y., Li, S. P., Li, Y., and Li, Y. Y. 2019. Profiling of indole metabolic pathway in thermo-sensitive Bainong male sterile line in wheat (Triticum aestivum L.). Physiol. Mol. Biol. Plants 25:263-275.

Summers, R. W., and Brown, J. K. M. 2013. Constraints on breeding for disease resistance in commercially competitive wheat cultivars. Plant Pathol. 62: 115-121.

Sun, H., Hu, J., Song, W., Qiu, D., Cui, L., Wu, P., Zhang, H., Liu, H., Yang, L., Qu, Y., Li, Y., Li, T., Cheng, W., Zhou, Y., Liu, Z., Li, J., and Li, H. 2018. Pm61: A recessive gene for resistance to powdery mildew in wheat landrace Xuxusanyuehuang identified by comparative genomics analysis. Theor. Appl. Genet. 131:2085-2097.

Tan, C., Li, G., Cowger, C., Carver, B. F., and Xu, X. 2019. Characterization of Pm63, a powdery mildew resistance gene in Iranian landrace PI 628024 Theor. Appl. Genet. 132:1137-1144.

Van Ooijen, J. W. 2006. JoinMap ${ }^{\circledR}$ 4, software for the calculation of genetic linkage maps in experimental populations. Kyazma BV, Wageningen, the Netherlands.

Wang, Z., Li, H., Zhang, D., Guo, L., Chen, J., Chen, Y., Wu, Q., Xie, J., Zhang, Y., Sun, Q., Dvorak, J., Luo, M. C., and Liu, Z. 2015. Genetic and physical mapping of powdery mildew resistance gene MlHLT in Chinese wheat landrace Hulutou. Theor. Appl. Genet. 128:365-373.

Xu, H., Yi, Y., Ma, P., Qie, Y., Fu, X., Xu, Y., Zhang, X., and An, D. 2015. Molecular tagging of a new broad-spectrum powdery mildew resistance allele $P m 2 c$ in Chinese wheat landrace Niaomai. Theor. Appl. Genet. 128:2077-2084.

Xue, F., Wang, C., Li, C., Duan, X., Zhou, Y., Zhao, N., Wang, Y., and Ji, W. 2012. Molecular mapping of a powdery mildew resistance gene in common wheat landrace Baihulu and its allelism with Pm24. Theor. Appl. Genet. 125: $1425-1432$.

Yahiaoui, N., Srichumpa, P., Dudler, R., and Keller, B. 2004. Genome analysis at different ploidy levels allows cloning of the powdery mildew resistance gene Pm $3 b$ from hexaploid wheat. Plant J. 37:528-538.

Yan, J., Kandianis, C. B., Harjes, C. E., Bai, L., Kim, E. H., Yang, X., Skinner, D. J., Fu, Z., Mitchell, S., Li, Q., Fernandez, M. G. S., Zaharieva, M., Babu, R., Fu, Y., Palacios, N., Li, J., DellaPenna, D., Brutnell, T., Buckler, E. S., Warburton, M. L., and Rocheford, T. 2010. Rare genetic variation at Zea mays crtRB1 increases $\beta$-carotene in maize grain. Nat. Genet. 42:322-327.

Yao, G., Zhang, J., Yang, L., Xu, H., Jiang, Y., Xiong, L., Zhang, C., Zhang, Z., Ma, Z., and Sorrells, M. E. 2007. Genetic mapping of two powdery mildew resistance genes in einkorn (Triticum monococcum L.) accessions. Theor Appl. Genet. 114:351-358.

Zhang, D., He, J., Huang, L., Zhang, C., Zhou, Y., Su, Y., and Li, S. 2017. An advanced backcross population through synthetic octaploid wheat as a "bridge": development and QTL detection for seed dormancy. Front. Plant Sci. 8:2123.

Zhang, P., Li, X., Takele-Weldu, G., Liu, H., Xia, X., He, Z., Li, Z., and Liu, D. 2019. QTL mapping of adult-plant resistance to leaf and stripe rust in wheat cross SW 8588/Thatcher using the wheat 55K SNP Array. Plant Dis. 103:3041-3049.

Zhang, R., Fan, Y., Kong, L., Wang, Z., Wu, J., Xing, L., Cao, A., and Feng, Y. 2018. Pm62, an adult-plant powdery mildew resistance gene introgressed from Dasypyrum villosum chromosome arm 2VL into wheat. Theor. Appl. Genet. 131:2613-2620.

Zhao, R., Liu, B., Jiang, Z., Chen, T., Wang, L., Ji, Y., Hu, Z., He, H., and Bie, T. 2019. Comparative analysis of genetic effects of wheat-Dasypyrum villosum translocations T6V\#2S·6AL and T6V\#4S·6DL. Plant Breed. 138:503-512.

Zou, S., Wang, H., Li, Y., Kong, Z., and Tang, D. 2018. The NB-LRR gene Pm60 confers powdery mildew resistance in wheat. New Phytol. 218:298-309. 